\title{
Das Amazonasbecken: Eine vergessene Kulturlandschaft und ihre Medizin
}

\begin{abstract}
The Amazon Basin: A Forgotten Cultural Landscape and Its Medicine. While the Amazon region's ecological importance remains uncontested, its role as a cultural hotspot is largely unknown to most people. Yet, recent archeological findings revise the image of a lush but inhospitable landscape whose farm produce could not sustain advanced civilization. The indigenous people today are only a tiny remainder of a far bigger population that developed impressive agricultural and forest engineering skills - until it was wiped out by diseases brought in from Europe. In fact, modern medicine benefits greatly from biological knowledge of indigenous Amazonians even today. This resource could prove to be much more valuable than any short-term profit realized by slash-and-burn farming or the extraction of raw materials. Therefore, it is all the more important to protect this endangered region. Scientific research will not only help to rescue indigenous biomedical knowledge, it will also give back respect and dignity to socalled savages and their cultural achievements.
\end{abstract}

\section{Das Amazonasgebiet als Wüste in Grün}

Unser Planet beherbergt eine Fülle an Naturregionen, denen der Mensch nur mit Ehrfurcht und Andacht begegnen kann. Von diesen ist das Amazonasbecken erstaunlich tief im kulturellen Gedächtnis des Globalen Nordens verankert, Amazonien ist zum Mythos geworden (Fatheuer 2019, S. 11). Diese starke Präsenz überrascht, denn im Gegensatz zu anderen Landschaften eignete sich diese nur bedingt als touristischer Sehnsuchtsort. Es fehlt darüber hinaus die ökonomische Bedeutung - wenn man einmal von dem vergleichsweise kurzen Kautschukboom absieht, der etwa 1870 begann und bereits 1915 abebbte. Der Amazonas fand Eingang in Weltliteratur und Filmkunst, jedoch in Maßen, nicht als unendlich zum Klischee perpetuierter Schauplatz. In unserer Vorstellungswelt oszilliert der Amazonas zwischen „Lunge der Welt“ und „Grüne Hölle“, zwischen Piranhas und Curare.

Roman M. Marek, Interdisziplinäre Arbeitsgruppe „Zukunft der Medizin: Gesundheit für alle“, Berlin-Brandenburgische Akademie der Wissenschaften

Ә OpenAccess. (C) 2021 Roman M. Marek, publiziert von De Gruyter. (cc) BY-NC-SA Dieses Werk ist lizenziert unter einer Creative Commons Namensnennung - Nicht kommerziell - Weitergabe unter gleichen Bedingungen 4.0 International Lizenz. https://doi.org/10.1515/9783110713336-014 
Vielleicht hat es sich als schicksalshaft erwiesen, dass ein derart exotisches Gebiet durch seine Namensgebung dann doch wieder Anknüpfung fand an die reichhaltige Welt griechischer Mythen; ${ }^{1}$ und damit an das, was wir gemeinhin als die Wiege unserer ,abendländischen Kultur“ verstehen. Als der spanische Eroberer Francisco de Orellana Bejarano Pizarro y Torres de Altamirano (1511-1546) im Jahr 1541 als erster Europäer den Amazonas bereiste, traf er dort - so sein Bericht - auf hellhäutige Kriegerinnen, die sich durch besonderen Mut, aber auch durch Kampfeslust und Wildheit hervortaten (Markham 2010 [1859]). Allem Anschein nach hinterließ diese radikale Umkehr gewohnter Rollenbilder einen bleibenden Eindruck, denn ganz im Bann ihrer von europäischen Werten, europäischen Mythen und der europäischen Geschichte ausgehenden Weltsicht benannten sie den Fluss just nach diesen wilden „Amazonen“.

Wie wir noch sehen werden, bestimmen diese beiden Topoi - Eurozentrismus (Chakrabarty 2000; Quijano 2002) und Wildheit - unsere Denkmuster bis zum heutigen Tag.

Mit einer Fläche von etwa sieben Millionen Quadratkilometern ist das Amazonasbecken größer als ganz Europa ohne Russland. In dieser unermesslichen Weite wurde es nicht nur zum Auffangbecken für etwa 15\% des Süßwassers, das sich weltweit in die Ozeane ergießt, sondern auch für alles, was das Gegenteil von „uns“ ist: hier Kultur, dort Natur; hier Zivilisation, dort unberührte Wildnis; hier fruchtbares Ackerland, dort nährstoffarme Böden; hier prosperierende Städte, dort Menschenleere. Unstrittig ist, dass der Amazonas-Regenwald die größte Biodiversität hat (Ter Steege et al. 2016) und eine der wichtigsten Stützen des Weltklimas ist (Nepstad et al. 2008): „a pillar of life on Earth“ (World Wide Fund For Nature 2020).

1 Die Etymologie des Begriffs „Amazone“ ist noch nicht eindeutig geklärt (Blok 1994, S. 21-37). Doch unabhängig davon, ob es die Amazonen tatsächlich gab, galten sie der antiken griechischen Welt als Prototyp des Kämpferisch-Wilden und Anderen, das sich nicht in ihre Welt integrieren ließ: „As autonomous women warriors, they were reputed to have founded a society of their own, where men were either absent or subservient. Hence the Amazons could not be accommodated in the regular realm of history, where men were unquestionably assumed to be the agents of culture and politics“ (Blok 1994, S. 433-432). 


\section{Der Amazonas als Kulturlandschaft: Neue Erkenntnisse aus Bioarchäologie und Paläoökologie}

Nur langsam dringt in das Bewusstsein der Weltöffentlichkeit, dass das Amazonasgebiet bei aller Biodiversität auch eine Kulturlandschaft ist: Hier lebt eine Vielzahl indigener Völker, die Europa in Anzahl, sowie sprachlicher und kultureller Diversität in nichts nachstehen. So gibt es über 350 ethnische Gruppen von denen etwa 60 noch weitgehend unberührt leben (World Wide Fund For Nature 2020). Jedoch gehören von den heute ca. 30 Millionen Bewohnern des Amazonasgebiets nur noch 9\% zur indigenen Bevölkerung, wobei die Grenzen zur mehrheitlichen Mestizen-Kultur fließend sind. Bis heute hält sich deshalb das Bild einer kleinen, einfachen, durch ihre natürliche Umgebung stark eingeschränkten und vereinzelt lebenden indigenen Bevölkerung (Clement et al. 2015; Heckenberger 2010), die im Gegensatz zu z.B. den mittelamerikanischen und andinen Hochkulturen keine nennenswerten zivilisatorischen Leistungen hervor gebracht hat.

Für die Archäologie stellt das Amazonasgebiet eine ganz besondere Herausforderung dar: Einerseits ist das Gebiet riesig und kaum erschlossen, andererseits tragen die klimatischen Bedingungen nicht gerade zum Erhalt organischer Materialien bei. Doch zwei sehr gegensätzliche Prozesse haben hier neue Forschungsmöglichkeiten eröffnet: Einerseits brachten die zunehmende wirtschaftliche Erschließung und die Abholzung unvorstellbar großer Flächen immer mehr Artefakte und Ruinen ans Licht (De Souza et al. 2018; Roosevelt 2013; Rostain 2008; Watling et al. 2017). Andererseits führten neue und verbesserte Methoden zu einer Blüte der Bioarchäologie und Paläoökologie. Beide Prozesse zusammen mündeten in den letzten 25 Jahren in Forschungsergebnisse, die im Hinblick auf die Betrachtung des Amazonasbeckens einen Paradigmenwechsel auslösten (Balée 2013; Clement et al. 2015; Erickson 2008; Pärssinen et al. 2020; Roosevelt 2013).

Die erste Überraschung war, dass Menschen bereits viel länger als ursprünglich gedacht dauerhaft im angeblich so unwirtlichen Amazonasbecken siedelten. In von den damaligen Bewohnern künstlich geschaffenen, verdächtig runden und noch heute sichtbaren „Waldinseln“ fanden sich Hinweise darauf, dass sich die ersten sesshaften Kulturen nicht wie zuvor angenommen erst um die Zeitenwende, sondern bereits vor 7.000, möglicherweise sogar 10.000 Jahren bildeten (Capriles et al. 2019). In einigen Gebieten wurde zudem eine verzweigte Raumordnung mit Grabenanlagen, Wällen und breiten Straßen sichtbar, die 
„Gartenstädte“ im Urwald miteinander verknüpften (prehistoric urbanism). Dies kann als Hinweis auf eine komplexere politische Gesellschaftsstruktur interpretiert werden (Heckenberger 2010).

Genetische Untersuchungen zeigten außerdem, dass einige der Völker des Amazonasbeckens eher mit den indigenen Einwohnern Australiens, Neu-Guineas und der Andamanen verwandt sind als mit anderen indigenen Amerikanern (Barbieri et al. 2019; Posth et al. 2018; Skoglund et al. 2015). Auch mit Polynesiern gab es präkolumbianische Kontakte (Ioannidis et al. 2020). Diese Erkenntnisse widersprechen der bisherigen „First Americans“-Hypothese, denn es musste mehrere Einwanderungswellen aus verschiedenen Richtungen gegeben haben. Bei der Untersuchung heutiger indigener Populationen gilt es zu bedenken, dass diese nur einen Bruchteil der ursprünglichen Bevölkerung vor Ankunft der Europäer ausmachen, die mit geschätzten acht Millionen etwa dreimal so hoch war wie heute (Clement et al. 2015).

Auch wenn diese Population nur etwa 10\% der damaligen Bevölkerungsdichte Europas ausmacht, ist sie für die nährstoffarmen Böden des Regenwaldes unerwartet groß. Zudem war sie nicht gleichmäßig über das Gebiet verteilt, es bildeten sich bedeutendere kulturelle Zentren (De Souza et al. 2018). Ein Beispiel dafür wäre der Chiribiquete National Park im Nordwesten des Amazonasbeckens mit der größten Fundstätte prähistorischer Felsmalereien Amerikas (UNESCO 2018). Diese neuen Erkenntnisse stützen die Berichte des eingangs erwähnten Konquistadoren Francisco de Orellana, die man zuvor als Übertreibung abgetan hatte. Darin beschreibt er nicht nur die so genannten „Amazonen“, sondern auch eine hohe Bevölkerungsdichte und größere Städte (Achmatowicz 2019; Harris 2017).

Ohne die Tötung, Versklavung, Kulturzerstörung und allgemeine Unterdrückung der indigenen Bevölkerung kleinreden zu wollen, waren es nach Ankunft der Europäer weniger diese selbst, als vielmehr die von ihnen eingeschleppten und dank Ansteckung sogar vorauseilenden Infektionskrankheiten, die den Großteil der einheimischen Bevölkerung auslöschten (Ashburn 1947; Chambouleyron et al. 2011; Cook 1998; Drake \& Oxenham 2013). Bereits eine der bis dato nicht vorkommenden Krankheiten (Pocken, Grippe, Masern, Mumps, Typhus, Malaria, Gelbfieber und Cholera) hätte zu einer tödlichen Epidemie geführt, doch die geballte Kombination gleich mehrerer Krankheitserreger rottete die immunologisch naive indigene Bevölkerung nahezu aus. Das gleiche Risiko besteht übrigens auch heute noch bei nicht kontaktierten Völkern (Pringle 2014). Neben den Epidemien hatten die Menschen damals zusätzlich noch mit einem länger anhaltenden Klimawandel und den dadurch ausgelösten Konflikten und Migrationsbewegungen zu kämpfen (De Souza et al. 2019). 
Dessen ungeachtet steht heute fest, dass im Amazonasbecken vor Ankunft der Europäer eine kulturell reichhaltige und diverse indigene Bevölkerung beachtliche zivilisatorische Leistungen vollbrachte: So wird das südwestliche Amazonasgebiet heute als eines der Hauptzentren landwirtschaftlicher Entwicklung gesehen (Browne Ribeiro 2017). Die Domestizierung und Züchtung von Nutzpflanzen markiert den Anfang jeglicher Hochkultur. Bisher hatte man hier weltweit vier Zentren ausgemacht: China (Reis), den Nahen Osten (Getreide und Hülsenfrüchte), Mittelamerika (Mais, Bohnen und Kürbisse), sowie die Andenregion (Kartoffeln und Quinoa). Dank neuer bioarchäologischer Erkenntnisse muss diesen etablierten Zentren nun ein fünftes hinzugefügt werden: Die südwestliche Amazonasregion (Lombardo et al. 2020).

Bereits im frühen Holozän, d.h. vor etwa 10.000 Jahren wurden in der Region Maniok und Kürbis kultiviert. Der erste Mais tauchte vor etwa 6.850 Jahren auf, außerdem wohl Erdnüsse, sowie einige Varianten von Chilis und Bohnen. Auch Kakao stammt nicht wie zuvor angenommen aus Mittelamerika, vielmehr begann die Kultivierung dieser Medizin- und Genusspflanze vor ca. 5.300 Jahren in der oberen Amazonasregion im heutigen südöstlichen Ecuador (Zarrillo et al. 2018). Tatsächlich gestaltete Menschen die Amazonasregion seit ihrer Ankunft um. Sie betrieben eine ganze eigene Form der Landwirtschaft, deren Spuren bis heute sichtbar sind. Die nur scheinbar unberührten Urwälder sind de facto über weite Strecken „Kulturwälder“ (cultural forests), geprägt durch technische Landschaftsplanung (Balée 2013).

Bei allen Debatten über Ausmaß und Verbreitung - einige Experten gehen von bis zu 12\% der Fläche des Regenwaldes aus - steht inzwischen fest, dass sich im Amazonasbecken eine Vielzahl heterogener, aber äußerst leistungsfähiger präkolumbianischer Kulturen entwickelte, die sich eng an ihre jeweilige Umwelt anpassten und diese sorgfältig bewirtschafteten und weiter entwickelten (heterogeneous landscape domestication) (Arroyo-Kalin 2010; De Souza et al. 2020; Erickson 2008; Harris 2017; Hermenegildo et al. 2017; Levis et al. 2018; Lombardo et al. 2020; Miller 2014; Odonne et al. 2019; Oliveira et al. 2020; Oliver 2008; Roosevelt 2013; Rostain 2008; Valdez 2003; Watling et al. 2017; Wingard \& Hayes 2013).

Mit zunehmender Abholzung zeigen sich auch die architektonischen Spuren dieser Amazonas-Kulturen. Vor etwa 10 Jahren entdeckte man große geometrische Strukturen aus Erdwällen, so genannte „Geoglyphen“, außerdem zeremonielle Zentren und ein entwickeltes Straßennetz (Pärssinen et al. 2020). Zudem entwickelte z.B. das Volk der Shipibo ein an eine eigene Schrift grenzendes Mustersystem. Das letzte der damit verfassten Bücher wurde erst 1978 verbrannt, angeblich enthielt es „Dinge des Teufels“ (Andritzky 1989, S. 190). Keine Hinweise fanden sich hingegen auf größere Abholzungen. Vielmehr schienen die Bewoh- 
ner bestimmte Pflanzen zu kultivieren, während sie gleichzeitig die anderen, ursprünglichen Pflanzen respektierten und an ihrem Platz beließen (Pärssinen et al. 2020).

\section{Erhöhung der Artenvielfalt durch indigene Land- und Forstwirtschaft}

Die Bewertung der Böden des Amazonas-Regenwaldes als unfruchtbar und nährstoffarm verrät den eurozentrischen Blick. Im Ergebnis eignet sich der Regenwald nicht für eine Landwirtschaft europäischer Prägung, die nach einer anfänglichen Brandrodung die mit anfälligen Monokulturen bepflanzten Böden schnell auslaugt. Im Gegensatz dazu erhöhen die landwirtschaftlichen Anbaumethoden der indigenen Kulturen die natürliche Vielfalt noch. Die ausgelaugte Erde des Regenwaldes reicherten sie mit Holzkohle und organischen Abfällen an, um so Bereiche mit fruchtbarer Schwarzerde (terra preta de índio) herzustellen (Arroyo-Kalin 2010; Levis et al. 2018; Oliveira et al. 2020). In eigentlich unbewohnbaren, saisonal überfluteten Savannenlandschaften schufen sie über hunderte von Kilometern erhöhte Felder (raised fields) (Rostain 2008) oder Waldinseln (forrest islands) (Lombardo et al. 2020; Watling et al. 2017).

In den so veränderten Landschaften erhöhte sich die Biodiversität, da in den kultivierten Bereichen andere Arten gedeihen als in den umgebenden, natürlich belassenen Gebieten. Die durch indigene Flächenbewirtschaftung (land management) kultivierten Bereiche erstrecken sich zum Teil über tausend Kilometer, und selbst 500 Jahre nach der letzten menschlichen Bearbeitung (die Bewohner verstarben nach Ankunft der Europäer) lässt sich die durch kulturelle Prozesse erhöhte Artenvielfalt noch immer nachweisen (Oliveira et al. 2020). Allem Anschein nach entwickelten die indigenen Völkern des Amazonasbeckens verschiedene besonders nachhaltige Techniken landwirtschaftlicher Bewirtschaftung, die eine sowohl Kultur als auch Natur bereichernde Koexistenz ermöglichten (Pärssinen et al. 2020).

\section{Indigene Konzepte von Gesundheit und Krankheit: Medizin- und Lehrpflanzen}

Diese erstaunliche kulturelle Leistung, die unserer eigenen Gesellschaft noch nicht gelungen ist, lässt auf eine besondere Geisteshaltung im Hinblick auf die natürliche Umgebung mit ihren Tieren, Pflanzen, Flüssen und Wäldern schließen, 
aus der eine ganz eigene Weltsicht mit reichhaltigen symbolischen Vorstellungen und rituellen Praktiken erwachsen ist. Diesem Selbstverständnis nach ist der Mensch eben nicht der Herr bzw. die Krone der Schöpfung. Bei allen kulturellen Unterschieden zwischen den indigenen Völkern ist ihnen doch gemeinsam, dass der Mensch eine eher untergeordnete Rolle spielt; nämlich die eines transienten Wesens, das erst noch werden und sich über sein soziales Gefüge und seine Rolle in der natürlichen Umwelt finden muss (Rival 2005).

Die Natur hingegen ist mit einem ganz eigenen Daseins- und Lebensrecht ausgestattet und tritt als des Menschen Lehrmeister in Erscheinung (Andritzky 1988, 1989; Jauregui et al. 2011). Grob zusammengefasst gibt es in den meisten metaphysischen Vorstellungen der indigenen Völker des Amazonasbeckens neben der Ebene des Hier-und-jetzt noch weitere Ebenen, z. B. die der Verstorbenen. Im Hier-und-jetzt tritt der Mensch nur als Gast auf, als Reisender, wobei die Pflanzen und Tiere die eigentlichen Bewohner dieser Ebene darstellen. Pflanzen und Tiere wird dabei meist eine eigene Persönlichkeit, Geschichte, und Sozialstruktur zugeschrieben. Prinzipiell sind aber die Grenzen fließend: Tiere können sich in Menschen verwandeln, und Menschen in Tiere (Viveiros de Castro 1998, 2012). Oftmals hat die Menschheit ihre Existenz stärkeren Naturkräften oder mythischen Tieren zu verdanken (z. B. Illius 1988). Tiere sind es auch, die in ihrer Agilität meist als Botschafter und Vermittler zwischen den Ebenen in Erscheinung treten. Die statischen Pflanzen hingegen werden meist als Träger uralter Kräfte und Weisheiten interpretiert, mit einem jeweils eigenen Charakter.

Diese Vorstellung zeigt sich auch im Begriff der Lehr- oder Meisterpflanzen (plantas maestras). Es ist nicht so, dass sich der Mensch dieser Pflanzen bedienen kann, etwa zu medizinischen Zwecken. Vielmehr verlangen die Pflanzen Respekt, Zuwendung und Aufmerksamkeit. Wer sich, etwa zu Heilzwecken, einer ritualisierten Pflanzenkur (dieta) unterwirft, muss fasten und im Hinblick auf viele Dinge des alltäglichen Lebens Verzicht üben (Jauregui et al. 2011). Durch die Einnahme pflanzlicher Extrakte wird dann eine Verbindung mit der Pflanze eingegangen, die für den Rest des Lebens Bestand haben soll. In der Vorstellung der indigenen Völker nimmt der Mensch die Pflanze, und mir ihr ihre Weisheit und Kraft in sich auf, so wie auch eine schwangere Frau ihr Kind in sich hat. Und dieses Kind gilt es zu nähren und zu respektieren damit eine lebenslängliche harmonische Verbindung aufgebaut werden kann (Andritzky 1988).

Auch die weiblichen oder männlichen Heiler (curanderos) ${ }^{2}$ sehen sich nicht als die Eigentümer oder Bewahrer des Wissens, vielmehr erhalten sie ihr Wissen

2 Der häufig anzutreffende Begriff „Schamane“ entstammt dem sibirisch-mongolisch-nordchinesischen Kulturkreis, entsprechend ist er mit ganz anderen Vorstellungen und Praktiken kon- 
von den Pflanzen und vermitteln lediglich zwischen den verschiedenen sozialen und spirituellen Entitäten. Während bei uns die Problembehandlung zwischen Ärzten, Psychologen, Richtern und Priestern etc. aufgeteilt wird, ist eine derartige Trennung zwischen körperlicher, geistig/emotionaler, sozialer und spiritueller Ebene der indigenen Vorstellungswelt fremd, denn eine Störung an einem Punkt würde sich automatisch auch auf alle anderen Bereiche auswirken (Rattay 2001).

\section{Beitrag der Amazonas-Medizin für die heutige medizinische Versorgung}

Die hier skizzierten Elemente indigener Weltvorstellungen beschränken sich sicherlich nicht auf das Amazonasgebiet - überall auf der Welt wird es vergleichbare Ansichten und holistische Gesundheitskonzepte geben. Das Besondere am Amazonasgebiet aber ist das Zusammenkommen dieses ganzheitlichen Konzeptes mit einer außergewöhnlich hohen Artenvielfalt und einer enormen kulturellen Diversität. Dennoch ist die Pflanzenmedizin des Amazonasbeckens beinahe unbekannt. Hinzu kommt, dass sie als das genaue Gegenteil westlicher Biomedizin gilt (Fotiou 2014). Während anderen Medizintraditionen wie etwa Āyurveda oder der Chinesischen Medizin noch eine gewisse Nähe - vielleicht Kultiviertheit zugebilligt wird, verkörpert dieses indigene medizinische Wissen das wilde, radikale Andere.

Tatsächlich aber hat die westliche Biomedizin längst indigenes medizinisches Wissen des Amazonasbeckens inkorporiert, das z. B. tagtäglich bei jeder Operation unter Narkose zum Einsatz kommt. So bildet die Anwendung von CurarePräparaten neben der Ausbildung der Intubationsnarkose und der Einführung kurzwirkender Narkotika eine der drei Grundlagen der modernen Anästhesie (Schmitt 1952). Curare, ein Sammelbegriff für die von den indigenen Völkern des Amazonasbeckens entwickelten Pfeilgifte, wurde erstmals von Columbus, anschließend aber auch von vielen anderen rückkehrenden Entdeckern beschrieben, wobei den Erzählungen immer auch ein Hauch der Verwunderung, des Geheimnisvollen und Unwahrscheinlichen anhaftete (Gicklhorn 1961; Hein 1981; Schmitt 1952).

Die wissenschaftliche Auseinandersetzung mit Curare in Europa begann erst im Jahr 1745 mit Charles Marie de La Condamine (1701-1774) in Leiden - wobei sich von diesem Punkt ausgehend erstmals ein internationaler Gedankenaus-

notiert und wird von den indigenen Völkern des Amazonasbeckens eher abgelehnt (The Shipibo Conibo Center of New York 2018). 
tausch über Experimentaltechniken und Wissensfortschritte entwickelte (Micheletti 2014). Curare ist demnach nicht nur als Wirkstoff bedeutend, viel mehr stellt dessen Untersuchung in einer Art Forschungsverbund auch den Anfang weltweiter Wissenschaftsentwicklung dar. Wichtige Beiträge zu diesen Anfängen pharmakologischer wissenschaftlicher Entwicklung leistete auch Alexander von Humboldt (1769-1859): In den Jahren 1799-1804 beschrieb er die Herstellung mehrerer Arten des Curare und unterschied sie anhand ihrer pharmakologischen Wirkung (Schmitt 1952).

Alexander von Humboldt berichtete neben Curare noch von einer weiteren Medizinzubereitung der indigenen Völker des Amazonasbeckens: Ayahuasca (Habekuß 2019; Ríos 1962). Ayahuasca ist ein Pflanzensud, dessen Hauptwirkstoffe $N, N$-Dimethyltryptamin (DMT) und verschiedene zu den $\beta$-Carbolinen gehörende Harman-Alkaloide sind (Simão et al. 2019). Interessant ist, dass beide Wirkstoffe aus zwei unterschiedlichen Pflanzen stammen: Die Harman-Alkaloide aus dem Stamm einer Liane (Banisteriopsis caapi), und das DMT aus den Blättern eines Busches (z. B. Psychotria viridis). DMT kommt über die ganze Welt verbreitet in verschiedenen Pflanzen vor, aber es wird auch endogen von verschiedenen Tieren (Menschen mit eingeschlossen) produziert (Cameron \& Olson 2018); in seiner chemische Struktur ähnelt es stark dem wichtigen Neurotransmitter Serotonin und dem Hormon Melatonin. Wird DMT oral aufgenommen, entfaltet es jedoch keine Wirkung, da es im Verdauungstrakt sehr schnell durch bestimmte Enzyme, die Monoaminooxidasen (MAO), abgebaut wird. Vor hunderten, vielleicht sogar tausenden von Jahren entdeckten die Indigenen Völker des Amazonasgebiets jedoch, dass Harman-Alkaloide die Monoaminooxidase reversibel hemmen, dadurch wird die zweite Komponente DMT vor dem Abbau geschützt und kann ihre pharmakologische Wirkung entfalten (dos Santos \& Hallak 2020). Angesichts der Artenvielfalt des Amazonasgebiets fällt es schwer, hier an einen Zufall zu glauben - schließlich muss der Sud aus dem Pflanzenmaterial auch noch in einer stundenlangen Prozedur zubereitet werden. Auch in diesem Fall müssen die indigenen Völker des Amazonasbeckens also als Entdecker der pharmakologischen Wirkung der Harman-Alkaloide gelten. In der heutigen Medizin werden sie und andere nach dem gleichen Prinzip wirkende Monoaminooxidase-Hemmer (MAO-Hemmer) zur Behandlung von Depressionen und Encephalitis, sowie bei Parkinson-Patienten eingesetzt. Der 1958 zugelassene MAOHemmer Iproniazid war das erste Antidepressivum überhaupt. 


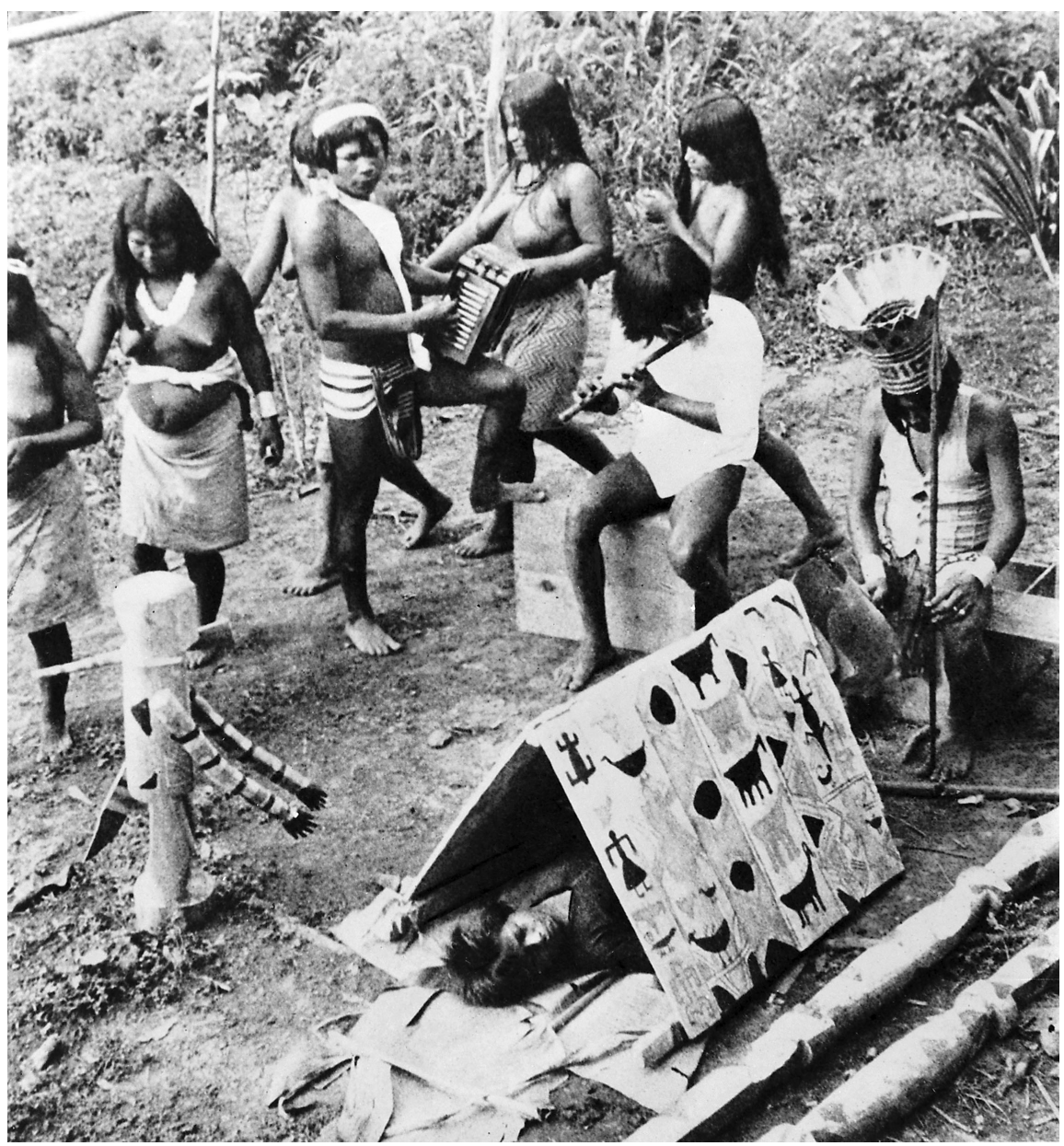

Abb. 1: Undatierte Aufnahme einer Heilzeremonie der Chocó-Indianer Kolumbiens, deren Lebensraum an das Amazonasbecken angrenzt. Der Patient liegt unter einem bemalten Zeremonialzelt, währen der Heiler (mit Federkrone) Beschwörungsformeln spricht. Im Hintergrund wird musiziert und getanzt. Links ist eine hölzerne Ritualfigur mit ausgestreckten Armen zu sehen, eventuell handelt es sich um ein Tier oder eine Ahnenfigur. Quelle: Wellcome Collection. Attribution 4.0 International (CC BY 4.0)

\section{Zukünftige Forschungsfelder: Psychopharmaka}

Traditionelle Medizin wird meist mit der Behandlung (kleinerer) körperlicher Leiden assoziiert. Doch wie die WHO in ihrer Definition regionaler Medizintra- 
ditionen festhält, können diese auch zur Prävention, Diagnose oder Behandlung psychischer Leiden zum Einsatz kommen (WHO 2020). Der indigenen AmazonasMedizin kommt auf diesem Gebiet eine besondere Rolle zu, denn zum Erhalt des psychisch-sozial-körperlichen Wohlergehens Einzelner oder der Gemeinschaft werden hier seit jeher spezielle Heilpraktiken eingesetzt. Dazu gehört auch das bereits erwähnte Ayahuasca, das bei zahlreichen indigenen Völkern ein integraler Bestandteil körperlicher, sozialer und spiritueller Heilriten ist: In ihren Augen handelt es sich um eine echte Medizin, die Respekt, Vorbereitung und die richtige Einstellung verlangt (Frecska et al. 2016; Salak 2006).

In den meisten Regionen des Globalen Südens gibt es keine Psychotherapeuten, sondern nur traditionelle Heiler, die Weltgesundheitsorganisation empfiehlt daher die weitere Erforschung und Qualitätssicherung regionaler Medizintraditionen, um diese in die allgemeine Gesundheitsversorgung $\mathrm{zu}$ integrieren (World Health Organization 2013, S. 43-57). Im Globalen Norden steigt unterdessen die Belastung durch psychische Störungen, es besteht ein großer Bedarf an neuen Therapiekonzepten und Behandlungsmöglichkeiten (Cressey 2011; Fibiger 2012; Pigott 2017). Interessanterweise reisen immer mehr Menschen aus dem Globalen Norden zu traditionellen Heilern in den Globalen Süden. In Peru und Brasilien z. B. floriert der Ayahuasca-Tourismus (Wolff et al. 2019). Gleichzeitig werden traditionelle Heiler nach Europa und Nordamerika eingeladen. So ist die Koexistenz verschiedener Medizinsysteme längst Realität geworden, der Amazonas ist gewissermaßen zu uns gekommen (ICEERS 2020).

Diese Situation sollte als Chance gesehen werden, denn die wissenschaftliche Auseinandersetzung mit der Pflanzenmedizin des Amazonasbeckens hat bisher äußerst vielversprechende Resultate erzielt. Für Ayahuasca etwa konnten teilweise deutlich positive Effekte auf psychische Leiden wie Depressionen, Substanzmissbrauch, sowie Ess- und Angststörungen nachgewiesen werden (Argento et al. 2019; Barbosa et al. 2018; Baumeister et al. 2014; Cruz \& Nappo 2018; Dos Santos \& Bouso 2019; dos Santos et al. 2016; Frecska et al. 2016; Hamill et al. 2019; Murphy-Beiner \& Soar 2020; Muttoni et al. 2019; Nunes et al. 2016; Palhano-Fontes et al. 2018; Renelli, Fletcher, Loizaga-Velder, et al. 2018; Renelli, Fletcher, Tupper, et al. 2018; Sanches et al. 2016).

Auch auf dem für eine alternde Bevölkerung so wichtigen Gebiet der chronischen neurodegenerativen Krankheiten (wie Demenz oder Alzheimer) könnte Ayahuasca eine Rolle spielen. Noch bis 1990 ging man davon aus, dass die neuronalen Verbindungen unseres Gehirns unveränderbar festgelegt sind und Regeneration unmöglich ist. Inzwischen konnte jedoch nachgewiesen werden, dass das menschliche Nervensystem ein geradezu erstaunliches Regenerationspotential aufweist (Neuroregeneration) (Sharma 2012). Dies hat zu einem neuen Forschungsfeld und letztlich zu einem Paradigmenwechsel geführt: weg von der 
Vorstellung eines statischen und hin zu einem lebenslang dynamisch modulierbaren Nervensystem. Verletzungen, Krankheiten und auch altersbedingte funktionelle Defizite können prinzipiell kompensiert werden, unser Gehirn kann sich anpassen (Bennett et al. 1964). Ziel aktueller Forschungsansätze ist es nun, die unserem Gehirn inhärente Fähigkeit zur Reorganisation zu modulieren und therapeutisch zu nutzen, d. h. Neuroplastizität zu fördern (Sasmita et al. 2018; Smith 2013). Der Stimulierung neurotropher Faktoren (NTF) durch psychoaktive Substanzen kommt dabei eine besondere Rolle zu (Frankel \& Cunningham 2002; Gewirtz et al. 2002; González-Maeso et al. 2007). In Zellkulturen und Tiermodellen konnte bereits gezeigt werden, dass die beiden Hauptkomponenten von Ayahuasca die Neuroplastizität erhöhen, so fördern Harman-Alkaloide und DMT die Neubildung und Vernetzung von Gehirnzellen (Dakic et al. 2016; Ly et al. 2018; Morales-García et al. 2020; Morales-García et al. 2017). Der traditionelle Gebrauch von Ayahuasca ließe sich heute mit modernen Forschungsergebnissen kombinieren, um so eine wissenschaftlich fundierte Anwendungsstrategie $\mathrm{zu}$ entwickeln.

Bisher jedoch konnte nur ein Bruchteil der seit Jahrhunderten angewendeten Medizinrituale erfasst und untersucht werden, dies gilt insbesondere für die Wirkmechanismen psychoaktiver Pflanzenzubereitungen (Cauper Pinedo 2018; Desmarchelier et al. 1996; Jauregui et al. 2011; Miller et al. 2019; Tournon \& Reátegui 1984; Tournon \& Silva 1988), neben Ayahuasca wären hier z. B. auch Sanaga oder aus Tieren gewonnene Wirkstoffe wie Kambô zu nennen (Hesselink \& Winkelman 2019; Majić et al. 2021; Schmidt et al. 2020). Gerade auf dem Gebiet der Psychopharmaka besteht ein hoher Bedarf an neuen Therapieoptionen, hier besitzt die traditionelle Pflanzenmedizin des Amazonasbeckens ein enormes und noch nicht erschlossenes Potential. Aus diesem Grund wird der Ruf immer lauter, die Forschung auf diesem Gebiet zu fördern und neue Konzepte für substanzunterstütze Psychotherapien zu untersuchen (Davis 2020; Schenberg 2018). Der Ruf ist umso dringlicher, denn aufgrund aktueller Entwicklungen ist die Welt der indigenen Völker des Amazonasbeckens eine Welt, die im Verschwinden begriffen ist (Ceballos Betancur \& Finger 2020).

\section{Gefährdung des Amazonasbeckens: Raubbau, Missionierung, Epidemien, Tourismus}

Für das Amazonasbecken brachte das Jahr 2020 eine existenzbedrohende Doppel-Krise: Einerseits nahmen Brandrodungen und Gebietsbesetzungen ein ungeahntes Ausmaß an, gleichzeitig war die indigene Bevölkerung der COVID-19- 
Pandemie größtenteils schutzlos ausgeliefert (Fischer 2020; Spektrum.de 2020). Im Schatten der Pandemie nahmen zudem die Attacken auf indigene Reservate zu (Bourscheit 2020). Die indigenen Völker erlebten die Pandemie als Retraumatisierung, als weitere eingeschleppte tödliche Epidemie, als Wiederkehr der Pocken (Madre Viruela). Sie zogen sich - wenn möglich - in entlegene Gebiete zurück und vertrauten für die Behandlung ihrer traditionellen Medizin, hier besteht die Befürchtung, dass kleinere Völker nun ganz aussterben könnten (Amigo 2020; Colmán Gutiérrez 2020).

Leider war das Amazonasgebiet bereits vor der COVID-19-Pandemie gefährdet. In seinem nachsynodalen apostolischen Schreiben Querida Amazonia äußerte sich Papst Franziskus besorgt:

\begin{abstract}
Bekanntlich wurde Amazonien [...] präsentiert, als wäre es einfach ein enormer leerer Raum, der besetzt, ein Reichtum im Rohzustand, der entwickelt, eine weite Wildnis, die gebändigt werden muss. All das im Zusammenhang mit einer Sichtweise, welche die Rechte der ursprünglichen Völker nicht anerkennt oder diese einfach ignoriert, als würde es sie gar nicht geben oder als würden ihnen die Gebiete, in denen sie wohnen, nicht gehören (Papst Franziskus 2020, S. 10).
\end{abstract}

Hier zeigt sich die fatale Wirkung des Topos der leeren Wildnis, der als implizite Rechtfertigung der zahlreichen Menschenrechtsverletzungen herhalten muss.

Neben der Bedrohung von Leib und Leben stehen die indigenen Völker des Amazonasbeckens allerdings auch vor der Bedrohung des Verlustes ihrer Kultur. $\mathrm{Zu}$ nennen wären an dieser Stelle besonders die evangelikalen Kirchen, die mit ihrer aggressiven Missionarstätigkeit ganze Gemeinschaften ihrer Kultur entfremden (Campos Lima 2019). Auf der anderen Seite sorgt auch der stark gestiegene Tourismus dafür, dass sich die indigene Kultur verfremdet, an den ausländischen Geschmack anpasst, und zum Souvenirkitsch verkommt (Fotiou 2014, 2016; Sánchez \& Bouso 2015; Stuart 2002; Wolff 2019). Viele indigene Gemeinschaften bemerken mit Sorge, wie traditionelle Heiler ihre Dörfer verlassen, um mit Touristen Geld zu verdienen, und wie medizinisches Wissen mitgenommen wird (spiritual extractivism) ohne den indigenen Menschen etwas zurück zu geben (The Shipibo Conibo Center of New York 2018). Eine weitere Gefahr besteht darin, dass sich der zunehmende Gesundheitstourismus auf die indigenen Heilmethoden auswirkt: Oft erwarten die angereisten Therapeuten und Klienten dann doch ein klassisches europäisches Heilungsmodell, das sich vor allem auf das Individuum zentriert, indigene Heiler passen sich dieser Erwartungshaltung dann aus kommerziellen Gründen an und erfinden dann eine „Tradition“, die einerseits von Reduktion und andererseits von Übertreibung geprägt ist (Wolff 2020, S. $234-$ 236). 


\section{Schlussfolgerungen: Forschung als Rettung und Anerkennung}

Es bleibt abzuwarten, ob von zahlreichen Prominenten unterstütze Appelle an die große Politik ihre Wirkung entfalten (Spiegel.de 2020). Im Hinblick auf die Umweltzerstörung könnte jeder Einzelne durch überlegtes Konsumverhalten zumindest einen kleinen Beitrag leisten (Rajão et al. 2020). Doch das medizinische Wissen der indigenen Völker des Amazonasbeckens ist eine Ressource, deren Erschließung für die Weltgemeinschaft viel wertvoller sein dürfte als die kurzfristigen Gewinne aus Brandrodung und Rohstoffabbau. Umso wichtiger ist es, dass die verbliebenen Reste dieser Region wirksam geschützt werden, und dass die darauf lebenden indigenen Völker anerkannt und ihre Rechte respektiert werden.

Bereits zum jetzigen Zeitpunkt hat unsere Gesellschaft dem biologischen Wissen und den Kulturleistungen der indigenen Völker des Amazonasbeckens viel zu verdanken - gerade auf dem Gebiet der Medizin. Eine dezidierte Förderung der wissenschaftlichen Erschließung der Pflanzen-Medizin des Amazonasbeckens würde einerseits medizinisches Wissen vor dem Verschwinden bewahren, und andererseits möglicherweise neue Therapieoptionen erschließen. Es besteht zudem die berechtigte Hoffnung, in der Auseinandersetzung mit indigenem Wissen neue Wege zu finden, die aufzeigen, wie auch wir im Globalen Norden eine friedliche Koexistenz von Biodiversität und Zivilisation entwickeln und ausbauen könnten (Institute of Social \& Cultural Anthropology 2020).

Die Auseinandersetzung mit den indigenen Völkern des Amazonasbeckens verspricht somit einen enormen Wissenszuwachs im Hinblick auf die gegenseitige Abhängigkeit von Menschen und ihrer Umwelt, auf die Entwicklung sozialer Identitäten, sowie auf die Beziehungen zwischen der materiellen, sozialen, und emotionalen Welt (Institute of Social \& Cultural Anthropology 2020). Doch die wissenschaftliche Erschließung z.B. indigener Medizinpraktiken dient nicht nur uns selbst, sie würde gleichzeitig den Völkern, die dieses Wissen ursprünglich erworben haben, Anerkennung und Respekt verschaffen. $\mathrm{Zu}$ viele Apelle sind bisher ungehört verhallt.

\section{Literatur}

Achmatowicz, Jerzy (2019): „La expedición de Francisco de Orellana y la exploración del río de Amazonas hasta su desembocadura en el Atlántico“. In: Studia Iberystyczne 17, S. 37-58. 
Amigo, Ignacio (2020): „For Brazil’s Indigenous Communities, Pandemic Revives Memories of Earlier Plagues“. https://www.sciencemag.org/news/2020/04/brazil-s-indigenouscommunities-pandemic-revives-memories-earlier-plagues, besucht am 15.4.2020.

Andritzky, Walter (1988): Schamanismus und rituelles Heilen im Alten Peru: Die Menschen des Jaguar (Bd. 1). Berlin: Clemens Zerling.

Andritzky, Walter (1989): „Ethnopsychologische Betrachtung des Heilrituals mit Ayahuasca (Banisteriopsis Caapi) unter besonderer Berücksichtigung der Piros (Ostperu)“. In: Anthropos 84(1/3), S. 177-201.

Argento, Elena/Capler, Rielle/Thomas, Gerald et al. (2019): „Exploring Ayahuasca-assisted Therapy for Addiction: A Qualitative Analysis of Preliminary Findings among an Indigenous Community in Canada“. In: Drug and Alcohol Review 38(7), S. 781-789.

Arroyo-Kalin, Manuel (2010): „The Amazonian Formative: Crop Domestication and Anthropogenic Soils“. In: Diversity 2(4), S. 473-504.

Ashburn, Percy Moreau (1947): The Ranks of Death: A Medical History of the Conquest of America. New York: Coward-McCann.

Balée, William (2013): Cultural Forests of the Amazon: A Historical Ecology of People and Their Landscapes. Tuscaloosa: The University of Alabama Press.

Barbieri, Chiara/Barquera, Rodrigo/Arias, Leonardo et al. (2019): „The Current Genomic Landscape of Western South America: Andes, Amazonia, and Pacific Coast“. In: Molecular Biology and Evolution 36(12), S. 2698-2713.

Barbosa, Paulo Cesar Ribeiro/Tófoli, Luís F./Bogenschutz, Michael P. et al. (2018): „Assessment of Alcohol and Tobacco Use Disorders Among Religious Users of Ayahuasca“. In: Frontiers in Psychiatry 9, S. 1-12.

Baumeister, David/Barnes, Georgina/Giaroli, Giovanni et al. (2014): „Classical Hallucinogens as Antidepressants? A Review of Pharmacodynamics and Putative Clinical Roles“. In: Therapeutic Advances in Psychopharmacology 4(4), S. 156-169.

Bennett, Edward L./Diamond, Marian C./Krech, David et al. (1964): „Chemical and Anatomical Plasticity of Brain: Changes in Brain through Experience, demanded by Learning Theories, are found in Experiments with Rats“. In: Science 146(3644), S. 610-619.

Blok, Josine H. (1994): The Early Amazons. Modern \& Ancient Perspectives on a Persistent Myth. Leiden: Brill.

Bourscheit, Aldem (2020): „Por qué el contexto es esencial para informar sobre el COVID-19 en comunidades indígenas“. International Center for Journalists. https://ijnet.org/es/story/ por-qu\%C3\%A9-el-contexto-es-esencial-para-informar-sobre-el-covid-19-en-comunidadesind\%C3\%ADgenas, besucht am 12.5.2020.

Browne Ribeiro, Anna T. (2017): „Rice Cultivation in Ancient Amazonia“. In: Nature Ecology \& Evolution 1(11), S. $1598-1599$.

Cameron, Lindsay P./Olson, David E. (2018): „Dark Classics in Chemical Neuroscience: N,N-Dimethyltryptamine (DMT)“. In: ACS Chemical Neuroscience 9(10), S. 2344-2357.

Campos Lima, Eduardo (2019): „Evangelical Missions a Major Threat to Amazon Culture, Catholic Leaders Say“. https://cruxnow.com/church-in-the-americas/2019/09/ evangelical-missions-a-major-threat-to-amazon-culture-catholic-leaders-say/, besucht am 3.9.2019.

Capriles, José M./Lombardo, Umberto/Maley, Blaine et al. (2019): „Persistent Early to Middle Holocene Tropical Foraging in Southwestern Amazonia“. In: Science Advances 5(4), S. eaav5449. 
Cauper Pinedo, Samuel (2018): „Estudio de plantas medicinales desde conocimientos shipibo. Masisea, Perú“. In: Ciencia y Desarrollo 21(2), S. 7.

Ceballos Betancur, Karin/Finger, Evelyn (2020): „Den Amazoniern droht ein Genozid“. Zeit Online. https://www.zeit.de/2020/22/indigene-voelker-coronavirus-amazonas-regenwaldlatainamerika/komplettansicht, besucht am 6.6.2020.

Chakrabarty, Dipesh (2000): Provincializing Europe. Postcolonial Thought and Historical Difference. Princeton, N.J.: Princeton University Press.

Chambouleyron, Rafael/Barbosa, Benedito Costa/Bombardi, Fernanda Aires et al. (2011): „,Formidable Contagion': Epidemics, Work and Recruitment in Colonial Amazonia (1660-1750)“. In: História, Ciências, Saúde-Manguinhos 18(4), S. 987-1004.

Clement, Charles R./Denevan, William M./Heckenberger, Michael J. et al. (2015): „The Domestication of Amazonia before European Conquest“. In: Proceedings of the Royal Society B: Biological Sciences 282(1812), S. 20150813.

Colmán Gutiérrez, Andrés (2020): „Pueblos indígenas se aíslan y acuden a la medicina ancestral ante la pandemia“. International Center for Journalists. https://ijnet.org/en/ node $/ 8258$ ?bclid= IWAR0GRT0t0fTKbuejfHmrd6yLA629Zm7lr01pilMMddRCKZEMkNM0Tre5FYQ, besucht am 19.6.2020.

Cook, Noble David (1998): Born to Die. Disease and New World Conquest, 1492-1650. Cambridge: Cambridge University Press.

Cressey, Daniel (2011): „Psychopharmacology in Crisis“. Nature. https://www.nature.com/ news/2011/110614/full/news.2011.367.html, besucht am 6.6.2020.

Cruz, J. I./Nappo, S. A. (2018): „Is Ayahuasca an Option for the Treatment of Crack Cocaine Dependence?“. In: J Psychoactive Drugs 50(3), S. 247-255.

Dakic, Vanja/Maciel, Renata de Moraes/Drummond, Hannah et al. (2016): „Harmine Stimulates Proliferation of Human Neural Progenitors“. In: PeerJ 4, S. e2727.

Davis, Nicola (2020): „Ease Rules on Research into Psychedelic Drugs, Urges David Nutt“. https://www.theguardian.com/politics/2020/apr/02/ease-rules-on-use-of-psychedelicdrugs-in-research-urges-david-nutt, besucht am 20.5.2020.

De Souza, Jonas Gregorio/Alcaina Mateos, Jonas/Madella, Marco (2020): „Archaeological Expansions in Tropical South America during the late Holocene: Assessing the Role of Demic Diffusion“. In: PLoS One 15(4), S. e0232367.

De Souza, Jonas Gregorio/Robinson, Mark/Maezumi, S. Yoshi et al. (2019): „Climate Change and Cultural Resilience in Late Pre-Columbian Amazonia“. In: Nature Ecology \& Evolution 3(7), S. $1007-1017$.

De Souza, Jonas Gregorio/Schaan, Denise Pahl/Robinson, Mark et al. (2018): „Pre-Columbian Earth-builders Settled along the Entire Southern Rim of the Amazon“. In: Nature Communications 9(1), S. 1125.

Desmarchelier, C./Gurni, A./Ciccia, G. et al. (1996): „Ritual and Medicinal Plants of the Ese'ejas of the Amazonian Rainforest (Madre de Dios, Perú)“. In: Journal of Ethnopharmacology 52(1), S. 45-51.

Dos Santos, R. G./Bouso, J. C. (2019): „Translational Evidence for Ayahuasca as an Antidepressant: What's Next?“. In: Braz J Psychiatry 41(4), S. 275-276.

dos Santos, Rafael G./Osório, Flávia L./Crippa, José Alexandre S. et al. (2016): „Antidepressive, Anxiolytic, and Antiaddictive Effects of Ayahuasca, Psilocybin and 
Lysergic Acid Diethylamide (LSD): A Systematic Review of Clinical Trials Published in the Last 25 Years“. In: Therapeutic Advances in Psychopharmacology 6(3), S. 193-213.

dos Santos, Rafael Guimarães/Hallak, Jaime Eduardo Cecilio (2020): „Therapeutic Use of Serotoninergic Hallucinogens: A Review of the Evidence and of the Biological and Psychological Mechanisms“. In: Neuroscience \& Biobehavioral Reviews 108, S. 423-434. Drake, A./Oxenham, M. (2013): „Disease, Climate and the Peopling of the Americas“. In: Historical Biology 25(5-6), S. 565-597.

Erickson, Clark L. (2008): „Amazonia: The Historical Ecology of a Domesticated Landscape“. In: Helaine Silverman/William H. Isbell (Hrsg.): The Handbook of South American Archaeology. New York, NY: Springer, S. 157-183.

Fatheuer, Thomas (2019): Amazonien heute. Eine Region zwischen Entwicklung, Zerstörung und Klimaschutz. Berlin: Heinrich-Böll-Stiftung, https://www.boell.de/sites/default/files/ amazonien_heute_kommentierbar.pdf, besucht am 05.05.2020.

Fibiger, H. Christian (2012): „Psychiatry, The Pharmaceutical Industry, and The Road to Better Therapeutics“. In: Schizophrenia Bulletin 38(4), S. 649-650.

Fischer, Lars (2020): „Amazonas-Waldbrände: Doppel-Krise durch Covid-19 und Feuer“. https:// www.spektrum.de/news/doppel-krise-durch-covid-19-und-feuer/1743176, besucht am 10.6.2020.

Fotiou, Evgenia (2014): „On the Uneasiness of Tourism“. In: Beatriz Caiuby Labate/Clancy Cavnar (Hrsg.): Ayahuasca Shamanism. In the Amazon and Beyond. Oxford: Oxford University Press, S. 159-181.

Fotiou, Evgenia (2016): „The Globalization of Ayahuasca Shamanism and the Erasure of Indigenous Shamanism“. In: Anthropology of Consciousness 27(2), S. 151-179.

Frankel, Paul S./Cunningham, Kathryn A. (2002): „The Hallucinogen d-Lysergic Acid Diethylamide (d-LSD) Induces the Immediate-early Gene c-Fos in Rat Forebrain“. In: Brain Research 958(2), S. 251-260.

Frecska, E./Bokor, P./Winkelman, M. (2016): „The Therapeutic Potentials of Ayahuasca: Possible Effects against Various Diseases of Civilization“. In: Front Pharmacol 7, S. 35.

Gewirtz, Jonathan C./Chen, Andrew C./Terwilliger, Rose et al. (2002): „Modulation of DOI-induced Increases in Cortical BDNF Expression by Group II mGlu Receptors“. In: Pharmacology Biochemistry and Behavior 73(2), S. 317-326.

Gicklhorn, Renée (1961): „Der erste wissenschaftliche Bericht über das Curare“. In: Beiträge zur Geschichte der Pharmazie 13(1), S. 4-5.

González-Maeso, Javier/Weisstaub, Noelia V./Zhou, Mingming et al. (2007): „Hallucinogens Recruit Specific Cortical 5-HT2 A Receptor-Mediated Signaling Pathways to Affect Behavior“. In: Neuron 53(3), S. 439-452.

Habekuß, Fritz (2019): „Andrea Wulf: ,Er wäre ziemlich geschockt““. https://www.zeit.de/2019/ 09/andrea-wulf-kunsthistorikerin-alexander-von-humboldt-naturforscher, besucht am 5.5.2020.

Hamill, Jonathan/Hallak, Jaime/Dursun, Serdar M. et al. (2019): „Ayahuasca: Psychological and Physiologic Effects, Pharmacology and Potential Uses in Addiction and Mental Illness“. In: Current Neuropharmacology 17(2), S. 108-128.

Harris, Mark (2017): „Revisiting First Contacts on the Amazon 1500-1562“. In: Tempo 23(3), S. 509-527. 
Heckenberger, Michael J. (2010): „Amazoniens Tropenwälder - Eine alte Kulturlandschaft?“. https://www.spektrum.de/magazin/amazoniens-tropenwaelder-eine-altekulturlandschaft/1017407, besucht am 2.5.2020.

Hein, Wolfgang-Hagen (1981): „Zum Curarebericht des Jesuitenpaters Franz Xaver Veigl“. In: Beiträge zur Geschichte der Pharmazie 31(11), S. 81-84.

Hermenegildo, Tiago/O’Connell, Tamsin C./Guapindaia, Vera L. C. et al. (2017): „New Evidence for Subsistence Strategies of Late Pre-colonial Societies of the Mouth of the Amazon Based on Carbon and Nitrogen Isotopic Data“. In: Quaternary International 448, S. $139-149$.

Hesselink, Jan M. Keppel/Winkelman, Michael (2019): „Vaccination with Kambo Against Bad Influences: Processes of Symbolic Healing and Ecotherapy“. In: The Journal of Transpersonal Psychology 51(1), S. 28-49.

ICEERS (2020): „The Role of Traditional Medicines in Global Mental Health“. International Center for Ethnobotanical Education, Research, and Service. https://www.iceers.org/therole-of-traditional-medicines-in-global-mental-health/?fbclid= IWAR33ImbPRYrXQqLdjuXYwEitJTyOc7r4WQMOPeREgeVyXeh2UKvvOkI5rRE, besucht am 2.5. 2020.

Illius, Bruno (1988): „Die ,Große Boa‘. Kunst und Kosmologie der Shipibo-Conibo“. In: Mark Münzel (Hrsg.): Die Mythen Sehen. Bilder und Zeichen vom Amazonas. Frankfurt am Main: Museum für Völkerkunde, S. 705-728.

Institute of Social \& Cultural Anthropology, University of Oxford (2020): „Amazonian Anthropology“. University of Oxford. https://www.isca.ox.ac.uk/amazonian-anthropology, besucht am 2.5.2020.

Ioannidis, Alexander G./Blanco-Portillo, Javier/Sandoval, Karla et al. (2020): „Native American Gene Flow into Polynesia Predating Easter Island Settlement“. In: Nature, S. 2-6.

Jauregui, X./Clavo, Z. M./Jovel, E. M. et al. (2011): „,Plantas con madre': Plants that Teach and Guide in the Shamanic Initiation Process in the East-Central Peruvian Amazon“. In: Journal of Ethnopharmacology 134(3), S. 739-752.

Levis, Carolina/Flores, Bernardo M./Moreira, Priscila A. et al. (2018): „How People Domesticated Amazonian Forests“. In: Frontiers in Ecology and Evolution 5, S. 1-21.

Lombardo, Umberto/Iriarte, José/Hilbert, Lautaro et al. (2020): „Early Holocene Crop Cultivation and Landscape Modification in Amazonia“. In: Nature 581(7807), S. 190-193.

Ly, Calvin/Greb, Alexandra C./Cameron, Lindsay P. et al. (2018): „Psychedelics Promote Structural and Functional Neural Plasticity“. In: Cell Reports 23(11), S. 3170-3182.

Majić, Tomislav/Sauter, Meike/Bermpohl, Felix et al. (2021): „Connected to the Spirit of the Frog: An Internet-based Survey on Kambô, the Secretion of the Amazonian Giant Maki Frog (Phyllomedusa bicolor): Motivations for Use, Settings and Subjective Experiences“. In: Journal of Psychopharmacology 35(4), S. 421- 436.

Markham, Clements R. (2010 [1859]): „The Voyage of Francisco De Orellana“. In: Clements R. Markham (Hrsg.): Expeditions into the Valley of the Amazons, 1539, 1540, 1639.

Cambridge: Cambridge University Press, S. 21-40.

Micheletti, Silvia (2014): Experimentalisierung und internationale Kommunikationen: Der Fall Curare. Stuttgart: Deutscher Apotheker Verlag.

Miller, Melanie J./Albarracin-Jordan, Juan/Moore, Christine et al. (2019): „Chemical Evidence for the Use of Multiple Psychotropic Plants in a 1,000-Year-old Ritual Bundle from South America“. In: Proceedings of the National Academy of Sciences 116(23), S. 11207-11212. 
Miller, Theresa L. (2014): „Valuing Varietal Diversity: Indigenous Canela Horticulture in Northeast Brazil“. In: Society, Biology \& Human Affairs 78, S. 62-82.

Morales-García, Jose A./Calleja-Conde, Javier/Lopez-Moreno, Jose A. et al. (2020): „N,N-dimethyltryptamine Compound found in the Hallucinogenic Tea Ayahuasca, Regulates Adult Neurogenesis in vitro and in vivo“. In: Translational Psychiatry 10(1), S. 1-14.

Morales-García, Jose A./De la Fuente Revenga, Mario/Alonso-Gil, Sandra et al. (2017): „The Alkaloids of Banisteriopsis Caapi, the Plant Source of the Amazonian Hallucinogen Ayahuasca, Stimulate Adult Neurogenesis in vitro“. In: Scientific Reports 7(1), S. 5309.

Murphy-Beiner, A./Soar, K. (2020): „Ayahuasca's ,Afterglow': Improved Mindfulness and Cognitive Flexibility in Ayahuasca Drinkers“. In: Psychopharmacology (Berl) 237(4), S. $1161-1169$.

Muttoni, Silvia/Ardissino, Maddalena/John, Christopher (2019): „Classical Psychedelics for the Treatment of Depression and Anxiety: A Systematic Review“. In: Journal of Affective Disorders 258, S. 11-24.

Nepstad, Daniel C./Stickler, Claudia M./Filho, Britaldo Soares et al. (2008): „Interactions among Amazon Land Use, Forests and Climate: Prospects for a Near-term Forest Tipping Point“. In: Philosophical Transactions of the Royal Society B: Biological Sciences 363(1498), S. $1737-1746$.

Nunes, Amanda A./dos Santos, Rafael G./Osório, Flávia L. et al. (2016): „Effects of Ayahuasca and Its Alkaloids on Drug Dependence: A Systematic Literature Review of Quantitative Studies in Animals and Humans“. In: Journal of Psychoactive Drugs 48(3), S. $195-205$.

Odonne, Guillaume/Bel, Martijn/Burst, Maxime et al. (2019): „Long-term Influence of Early Human Occupations on Current Forests of the Guiana Shield“. In: Ecology 100(10), S. e02806.

Oliveira, Edmar Almeida/Marimon-Junior, Ben Hur/Marimon, Beatriz Schwantes et al. (2020): „Legacy of Amazonian Dark Earth Soils on Forest Structure and Species Composition“. In: Global Ecology and Biogeography, S. 1-16.

Oliver, José R. (2008): „The Archaeology of Agriculture in Ancient Amazonia“. In: Helaine Silverman/William H. Isbell (Hrsg.): The Handbook of South American Archaeology. New York, NY: Springer, S. $185-216$.

Palhano-Fontes, Fernanda/Barreto, Dayanna/Onias, Heloisa et al. (2018): „Rapid Antidepressant Effects of the Psychedelic Ayahuasca in Treatment-resistant Depression: A Randomized Placebo-controlled Trial“. In: Psychological Medicine 49(4), S. 655-663.

Papst Franziskus (2020): Querida Amazonia. http://www.vatican.va/content/francesco/de/ apost_exhortations/documents/papa-francesco_esortazione-ap_20200202_queridaamazonia.html Pressemitteilung vom 02.02.2020.

Pärssinen, Martti/Ferreira, Evandro/Virtanen, Pirjo Kristiina et al. (2020): „Domestication in Motion: Macrofossils of Pre-Colonial Brazilian Nuts, Palms and Other Amazonian Planted Tree Species Found in the Upper Purus“. In: Environmental Archaeology 26(3), S. 309-322.

Pigott, Ed (2017): „The Crisis in Psychopharmacology Provides an Opportunity for Neuroregulation Treatments to Gain Widespread Acceptance“. In: NeuroRegulation 4(1), S. $28-36$.

Posth, Cosimo/Nakatsuka, Nathan/Lazaridis, losif et al. (2018): „Reconstructing the Deep Population History of Central and South America“. In: Cell 175(5), S. 1185-1197.e1122. 
Pringle, Heather (2014): „Members of Previously Uncontacted Tribe Infected with Flu“. https:// www.sciencemag.org/news/2014/07/members-previously-uncontacted-tribe-infected-flu\#, besucht am 2.5.2020.

Quijano, Anibal (2002): „Colonialidad del Poder, eurocentrismo y America Latina“. In: Edgardo Lander (Hrsg.): La Colonialidad del saber: eurocentrismo y ciencias sociales. Perspectivas latinoamericanas. Buenos Aires: CLACSO, S. 201-246.

Rajão, Raoni/Soares-Filho, Britaldo/Nunes, Felipe et al. (2020): „The Rotten Apples of Brazil's Agribusiness“. In: Science 369(6501), S. 246.

Rattay, Brigitta (2001): „Susto und Curanderismo - ein holistisches und anpassungsfähiges Medizinmodell“. In: Curare. Zeitschrift für Ethnomedizin und Transkulturelle Psychiatrie 24(1), S. 65-80.

Renelli, Marika/Fletcher, Jenna/Loizaga-Velder, Anja et al. (2018): „Ayahuasca and the Healing of Eating Disorders“. In: Hillary L. McBride/Janelle L. Kwee (Hrsg.): Embodiment and Eating Disorders. Theory, Research, Prevention and Treatment. New York: Routledge, S. $214-230$.

Renelli, Marika/Fletcher, Jenna/Tupper, Kenneth W. et al. (2018): „An Exploratory Study of Experiences with Conventional Eating Disorder Treatment and Ceremonial Ayahuasca for the Healing of Eating Disorders“. In: Eating and Weight Disorders - Studies on Anorexia, Bulimia and Obesity 25(2), S. 437-444.

Ríos, Oscar (1962): „Aspectos preliminares al estudio fármaco-psiquiátrico del Ayahuasca y su principio activo“. In: Anales de la Facultad de Medicina 45(1-2), S. 22-66.

Rival, Laura (2005): „Introduction: What Constitutes a Human Body in Native Amazonia?“. In: Tipití: Journal of the Society for the Anthropology of Lowland South America 3(2), S. $105-110$.

Roosevelt, Anna C. (2013): „The Amazon and the Anthropocene: 13,000 Years of Human Influence in a Tropical Rainforest“. In: Anthropocene 4, S. 69-87.

Rostain, Stéphen (2008): „Agricultural Earthworks on the French Guiana Coast“. In: Helaine Silverman/William H. Isbell (Hrsg.): The Handbook of South American Archaeology. New York, NY: Springer, S. 217-233.

Salak, Kira (2006): „Hell and Back“. In: National Geographic Adventure 8(2), S. 54-64.

Sanches, Rafael Faria/De Lima Osório, Flávia/Dos Santos, Rafael G. et al. (2016): „Antidepressant Effects of a Single Dose of Ayahuasca in Patients with Recurrent Depression“. In: Journal of Clinical Psychopharmacology 36(1), S. 77-81.

Sánchez, Constanza/Bouso, Carlos (2015): „Ayahuasca: From the Amazon to the Global Village. An Analysis of the Challenges Associated with the Globalisation of Ayahuasca“. Amsterdam: Transnational Institute.

Sasmita, Andrew Octavian/Kuruvilla, Joshua/Ling, Anna Pick Kiong (2018): „Harnessing Neuroplasticity: Modern Approaches and Clinical Future“. In: International Journal of Neuroscience 128(11), S. 1061-1077.

Schenberg, Eduardo Ekman (2018): „Psychedelic-Assisted Psychotherapy: A Paradigm Shift in Psychiatric Research and Development“. In: Frontiers in Pharmacology 9, S. 733.

Schmidt, Timo Torsten/Reiche, Simon/Hage, Caroline L. C. et al. (2020): „Acute and subacute psychoactive effects of Kambô, the secretion of the Amazonian Giant Maki Frog (Phyllomedusa bicolor): retrospective reports“. In: Scientific Reports 10(1), S. 1-11. 
Schmitt, Anton (1952): „Intubationsnarkose und Curareanwendung in der modernen

Anästhesie“. In: Karl Heinricht Bauer/Alfred Brunner (Hrsg.): Ergebnisse der Chirurgie und Orthopädie. Berlin \& Heidelberg: Springer, S. 436-500.

Sharma, Hari Shanker (2012): „Preface“. In: International Review of Neurobiology 102, S. xv-xx. Simão, Ana Y./Gonçalves, Joana/Duarte, Ana Paula et al. (2019): „Toxicological Aspects and Determination of the Main Components of Ayahuasca: A Critical Review“. In: Medicines (Basel) 6(4), S. 106.

Skoglund, Pontus/Mallick, Swapan/Bortolini, Maria Cátira et al. (2015): „Genetic Evidence for Two Founding Populations of the Americas“. In: Nature 525(7567), S. 104-108.

Smith, Gwenn E. (2013): „Introduction“. In: Intersections: Canadian Journal of Music 33(2), S. 3.

Spektrum.de (2020): „Rekordabholzung im Schatten der Coronakrise“. https://www.spektrum. de/news/rekordabholzung-im-schatten-der-coronakrise/1722314, besucht am 2.5.2020.

Spiegel.de (2020): „Offener Brief an Bolsonaro. Stars fordern Schutz für Brasiliens Ureinwohner“. https://www.spiegel.de/panorama/leute/brad-pitt-madonna-und-paulmccartney-stars-fordern-schutz-vor-coronavirus-fuer-ureinwohner-brasiliens-a-9a2f26875a06-4c0c-abb1-70fba52b39e5, besucht am 5.5.2020.

Stuart, R. (2002): „Ayahuasca Tourism: A Cautionary Tale“. In: Newsletter of the Multidisciplinary Association for Psychedelic Studies MAPS 12(2), S. 36-38.

Ter Steege, Hans/Vaessen, Rens W./Cárdenas-López, Dairon et al. (2016): „The Discovery of the Amazonian Tree Flora with an Updated Checklist of All Known Tree Taxa“. In: Scientific Reports 6(1), S. 29549.

The Shipibo Conibo Center of New York (2018): „In the Declaration of Yarinacocha, Shipibo Healers Organize To Resist Spiritual Extractivism“. https://realitysandwich.com/323245/ in-the-declaration-of-yarinacocha-shipibo-healers-organize-to-resist-spiritual-extractivism/ ?fbclid=IwAR3Q2HxDpw1EubbCBsXQaJ-VflDbOjrgxVerFoGRBtjzfsrl3cjYtjeC67 g, besucht am 5.5.2020.

Tournon, Jacques/Reátegui, U. (1984): „Investigaciones sobre las plantas medicinales de los Shipibo-Conibo del Ucayali“. In: Amazonía Peruana 5(10), S. 91-118.

Tournon, Jacques/Silva, Milton (1988): „Plantas para cambiar el comportamiento humano entre los shipibo-conibo“. In: Anthropologica Del Departamento De Ciencias Sociales 6(6), S. 161-176.

UNESCO (2018): „Chiribiquete National Park - ,The Maloca of the Jaguar““. https://whc.unesco. org/en/list/1174/, besucht am 5.5.2020.

Valdez, Francisco (2003): Primeras sociedades de la alta Amazonia: La cultura Mayo Chinchipe-Marañón. Marseille: IRD Éditions.

Viveiros de Castro, Eduardo (1998): „Cosmological Deixis and Amerindian Perspectivism“. In: The Journal of the Royal Anthropological Institute 4(3), S. 469-488.

Viveiros de Castro, Eduardo (2012): „Cosmological Perspectivism in Amazonia and Elsewhere. Four Lectures Given in the Department of Social Anthropology, University of Cambridge, February-March 1998“. In: HAU Masterclass Series 1, S. 45-82.

Watling, Jennifer/Iriarte, José/Mayle, Francis E. et al. (2017): „Impact of Pre-Columbian ,Geoglyph“ Builders on Amazonian Forests“. In: Proceedings of the National Academy of Sciences 114(8), S. 1868-1873. 
WHO (2020): „Traditional, Complementary and Integrative Medicine“. https://www.who.int/ health-topics/traditional-complementary-and-integrative-medicine\#tab=tab_1, besucht am 5.5.2020.

Wingard, John D./Hayes, Sue Eileen (2013): Soils, Climate and Society: Archaeological Investigations in Ancient America. Boulder: University of Colorado Press.

Wolff, Tom John (2019): „Ayahuasca-Tourismus in Südamerika“. In: Robert Feustel/Henning Schmidt-Semisch/Ulrich Bröckling (Hrsg.): Handbuch Drogen in sozial- und kulturwissenschaftlicher Perspektive. Wiesbaden: Springer VS, S. 555-575.

Wolff, Tom John (2020): The Touristic Use of Ayahuasca in Peru. Expectations, Experiences, Meanings and Subjective Effects. Wiesbaden: Springer VS.

Wolff, Tom John/Ruffell, Simon/Netzband, Nigel et al. (2019): „A Phenomenology of Subjectively Relevant Experiences Induced by Ayahuasca in Upper Amazon Vegetalismo Tourism“. In: Journal of Psychedelic Studies 3(3), S. 295-307.

World Health Organization (2013): WHO Traditional Medicine Strategy: 2014-2023. Geneva: World Health Organization.

World Wide Fund For Nature (2020): „Inside the Amazon“. https://wwf.panda.org/knowledge_ hub/where_we_work/amazon/about_the_amazon/, besucht am 5.5.2020.

Zarrillo, Sonia/Gaikwad, Nilesh/Lanaud, Claire et al. (2018): „The Use and Domestication of Theobroma Cacao during the Mid-Holocene in the Upper Amazon“. In: Nature Ecology \& Evolution 2(12), S. 1879-1888. 